Descripción de cultivar

\title{
Jade: nueva variedad de avena para la producción de grano en siembras de temporal en México
}

\author{
Héctor Eduardo Villaseñor Mir ${ }^{1}$ \\ Eduardo Espitia Rangel ${ }^{1}$ \\ María Florencia Rodríguez García ${ }^{1}$ \\ Eliel Martínez Cruz ${ }^{1 \S}$ \\ Julio Huerta Espino ${ }^{1}$ \\ René Hortelano Santa Rosa ${ }^{1}$ \\ Leodegario Osorio Alcalá ${ }^{2}$
}

${ }^{1}$ Campo Experimental Valle de México-INIFAP. Carretera Los Reyes-Texcoco km 13.5, Coatlinchán, Texcoco, Estado de México, México. (villasenor.hector@inifap.gob.mx; espitia.eduardo@inifap.gob.mx; rodriguez.maria@inifap.gob.mx; huerta.julio@inifap.gob.mx; hortelano.rene@inifap.gob.mx). ${ }^{2}$ Campo Experimental Valles Centrales de Oaxaca-INIFAP. Melchor Ocampo núm. 7, Santo Domingo, Barrio Bajo, Etla, Oaxaca, México. (osorio.leodegario@inifap.gob.mx).

${ }^{\S}$ Autor para correspondencia: martinez.eliel@inifap.gob.mx.

\section{Resumen}

El Instituto Nacional de Investigaciones Forestales Agrícolas y Pecuarias, pone a disposición de los productores de avena de México la variedad 'Jade', que fue obtenida por su Programa de Mejoramiento Genético ubicado en el Campo Experimental Valle de México (CEVAMEX). Jade se obtuvo a través de una cruza simple entre la línea 815A-129-72-CI-648/SR-CPX y la variedad Obsidiana, y su $F_{1}$ que se retrocruzó hacia la variedad, en la selección hacia homocigosis se aplicaron los métodos gravimétricos y familias masivas hasta obtener la línea experimental que se evaluó en 105 localidades en 12 estados de la república en condiciones de temporal de 2008 a 2012. Durante este periodo en JADE se registraron lecturas de roya del tallo de trazas (TR) a $20 \mathrm{MR}$, tipo de reacción de resistencia que superó a todas las variedades testigo. Su rendimiento de grano promedio fue de $3681 \mathrm{~kg} \mathrm{ha}^{-1}$ en ambientes favorables, $2302 \mathrm{~kg} \mathrm{ha}^{-1}$ en ambientes intermedios y $1382 \mathrm{~kg} \mathrm{ha}^{-1}$ en ambientes críticos, superando a las once variedades testigos en cada condición. En base a su comportamiento agronómico y resistencia a roya del tallo, con la siembra de Jade se puede sustituir a las variedades Menonita, Avemex, Papigochi, Chihuahua, Cuauhtémoc y Ópalo, lo que permitirá incrementar en promedio $28 \%$ la productividad de avena en siembras de temporal. Por lo anterior, Jade formará parte del mosaico genético de variedades que INIFAP recomienda para siembra de temporal en México.

Palabras clave: Avena sativa L., ambientes de temporal, mayor rendimiento, tolerancia a roya del tallo.

Recibido: julio de 2019

Aceptado: agosto de 2019 
En México durante 2017 la superficie sembrada con el cultivo de avena (Avena sativa L.) fue de 856354 ha, figurando en el quinto lugar con mayor superficie sembrada de los cultivos en nuestro país. El 94\% de la producción de avena fue en condiciones de temporal, 87\% fue para la producción de forraje en verde, henificado o en grano, se sembró en 21 entidades y destacaron por su importancia Chihuahua, Durango, Zacatecas, Estado de México e Hidalgo (SIAP, 2017).

Una limitante en la producción del cultivo de avena de temporal es la pérdida en rendimiento causada por la enfermedad roya del tallo (Puccinia graminis f. sp. avenae E. y H.), que en variedades susceptibles llega al 70\% (Leyva et al., 2004) y además, ocasiona la obtención de forraje de mala calidad (Espitia et al., 2012). Dado que su control químico es costoso por el número de aplicaciones necesarias, la forma más efectiva de reducir esas pérdidas es mediante la siembra de variedades resistentes o tolerantes a esta enfermedad (Villaseñor et al., 2009a). Adicionalmente, la sequía es un problema importante en el cultivo de avena que cada vez se acentúa más en las siembras de temporal en las diferentes regiones productoras, de tal manera, que las variedades precoces y con buena respuesta al estrés hídrico es la estrategia más adecuada para disminuir las pérdidas (Villaseñor et al., 2009b).

El mejoramiento genético de la avena en México, que ha sido responsabilidad del programa del INIFAP-CEVAMEX (Villaseñor et al. (2009b), se ha enfocado principalmente en la liberación de variedades que conjunten precocidad y tolerancia a la sequía y a la roya del tallo, así como mayor rendimiento de grano. Resultado del proceso de mejoramiento genético, el INIFAP pone a disposición de los productores de avena de México la nueva variedad Jade, para enfrentar la problemática biótica y abiótica de las zonas aveneras del país e incrementar su productividad. Jade se registró en el Catalogo de Variedades Factibles de Certificación del Servicio Nacional de Inspección y Certificación de Semillas con el número AVE-020-290514.

\section{Obtención de la variedad y características}

La variedad de avena Jade fue obtenida por recombinación genética, selección y evaluación por el Programa de Mejoramiento Genético de Avena del Instituto Nacional de Investigaciones Forestales Agrícolas y Pecuarias (INIFAP) con sede en el Campo Experimental Valle de México (CEVAMEX).

La cruza se realizó durante el ciclo otoño-invierno 1998-1999 (O-I/1998-99) en Chapingo, Estado de México. (CEVAMEX), participando en su cruzamiento como progenitor hembra línea experimental 815A-129-72-CI-648/SR-CPX y como macho la variedad Obsidiana, en el ciclo primavera-verano de 1999 (P-V/1999) en el CEVAMEX su $\mathrm{F}_{1}$ se retrocruzó con Obsidiana $\left(\mathrm{F}_{1} \mathrm{R}\right)$. La semilla de su $F_{1} R$ se sembró en el ciclo O-I/1999-2000 en el CEVAMEX y la población se cosechó masivamente. La generación $\mathrm{F}_{2}$ también se evaluó en esa estación experimental del INIFAP durante el ciclo P-V/2000, en donde la población núm. 4537 se sembró bajo competencia, se cosechó masivamente y posteriormente se le aplicó selección por densidad y peso de grano (0C). En la generación $\mathrm{F}_{3}$ sembrada en el CEVAMEX en el ciclo P-V/2001, se realizó selección individual de plantas (selección de la planta 5C) para derivar familias masivas en la generación $\mathrm{F}_{4}$, la cual fue sembrada en el Campo Experimental Bajío (CEBAJ) en Roque, Guanajuato. Durante el ciclo otoño-invierno (O-I) de 2001-2002 y la familia se cosechó masivamente (0R); este procedimiento también se practicó en la generación $\mathrm{F}_{5}$ en el CEVAMEX en el ciclo primaveraverano $(\mathrm{P}-\mathrm{V}) / 2002$, donde también la familia se cosechó masivamente (0C). 
La generación $\mathrm{F}_{6}$ se evaluó nuevamente en el CEVAMEX en el ciclo P-V/2003, en donde se realizó selección individual de plantas (selección de la planta 2C) para derivar líneas en la generación $\mathrm{F}_{7}$, semilla que fue sembrada en el CEBAJ en el ciclo O-I/2003-04 y la línea se cosechó masivamente (0R). En el ciclo P-V/2010 en la localidad de Coatepec, municipio de Ixtapaluca, Estado de México, se observó segregación en la línea experimental, por lo que se procedió hacer selección individual de 50 panículas, que fueron sembradas en el CEBAJ durante el ciclo O-I/2010-11, y en donde fue seleccionada masivamente la línea avanzada número 18 (18COAT-0R) que dio origen a Jade, la cual se identificó con los siguientes genealogía y pedigrí: 815A-129-72-CI-648/SRCPX)/2*OBSI-4537-0C-5C-0R-0C-2C-0R-18COAT-0R.

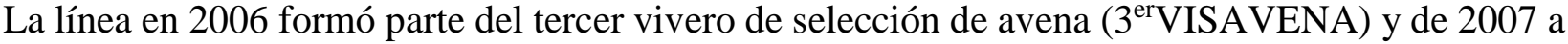
2012 formó parte del séptimo al doceavo ensayo nacional uniforme de avena ( $7^{\circ}$ EUAVENA al $12^{\text {vo }}$ EUAVENA). Lo anterior, permitió evaluarla hasta en 105 localidades que se ubicaron en 12 estados de la república que comprendieron Oaxaca, Puebla, Tlaxcala, Hidalgo, Estado de México, Guanajuato, Michoacán, Jalisco, Aguascalientes, Zacatecas, Durango y Chihuahua. La nueva variedad Jade es de hábito de primavera, de ciclo vegetativo intermedio (107 días promedio a madurez fisiológica), con una altura de planta media (100 cm promedio), tolerante al acame y responde favorablemente en cualquier tipo de ambiente de producción bajo temporal.

\section{Reacción a enfermedades}

Durante su periodo de evaluación la variedad Jade fue resistente a moderadamente resistente a roya del tallo (Puccinia graminis $\mathrm{f}$. sp. avenae), resistente a roya de la corona o de la hoja $(P$. coronata) y tolerante al complejo de enfermedades foliares causado por Helminthosporium avenae (mancha foliar), Septoria avenae f. sp. avenae (mancha foliar) y Colletotrichum graminicola (antracnosis).

En el Cuadro 1 se presenta la reacción a enfermedades de Jade y de las variedades testigo evaluadas de 2008 a 2012 bajo condiciones de temporal. Jade registró lecturas de roya del tallo de trazas de resistencia (TR) a 20\% de infección de moderada resistencia (20MR), tipo de reacción que superó a todas las variedades testigo; Mariscal et al. (2011) reportan la evaluación ante 24 razas diferentes de roya del tallo de ocho de las variedades testigo contra las que se probó Jade y de acuerdo con sus resultados, se indica que la resistencia de Jade a roya del tallo posiblemente se deba a la acción conjunta de 2 a 3 genes de efectos aditivos.

Para el caso de roya de la corona o de la hoja, la nueva variedad también fue el genotipo con mayor resistencia, manifestando incidencias de trazas de resistencia (TR) a 10\% de infección de resistencia (10R), superior a las variedades testigo Turquesa y Karma, las cuales presentaron incidencias hasta de 50\% de susceptibilidad. La nueva variedad Jade igualó la tolerancia al complejo de enfermedades foliares de la mayoría de las variedades testigo y mostró mayor resistencia a estos patógenos que las variedades susceptibles Papigochi y Menonita.

La incidencia mayor del complejo de enfermedades foliares se presentó en localidades con precipitaciones superiores a los $600 \mathrm{~mm}$, considerados en temporal como ambientes lluviosos para el cultivo de avena. Por su tolerancia a estas enfermedades, Jade es buena opción para recomendarse en este tipo de ambientes junto con Turquesa y Obsidiana, principalmente. 
Cuadro 1. Características agronómicas y fitopatológicas de la nueva variedad Jade y variedades testigo en ambientes de temporal de 2008 a 2013.

\begin{tabular}{ccccccc}
\hline Variedad & DAM & AP $(\mathrm{cm})$ & Acame & RT & RC & Foliares \\
\hline Jade & 107 & 100 & MR & TR a 20MR & TR a 10R & $6 / 40(\mathrm{~T})$ \\
Turquesa & 107 & 97 & MR & 5MR a 40MS & 10R a 30MS & $6 / 40(\mathrm{~T})$ \\
Obsidiana & 108 & 97 & MR & 10MS a 60S & 20MS a 70S & $5 / 20(\mathrm{~T})$ \\
Karma & 107 & 95 & $\mathrm{R}$ & 10MS a 50S & 10R a 50S & $6 / 40(\mathrm{~T})$ \\
Avemex & 108 & 111 & $\mathrm{MS}$ & 20MS a 80S & 20MS a 70S & $6 / 40(\mathrm{~T})$ \\
Menonita & 103 & 93 & $\mathrm{R}$ & 10MS a 80S & 20MS a 70S & $7 / 60(\mathrm{MS})$ \\
Papigochi & 106 & 92 & $\mathrm{MR}$ & 5MR a 70S & 20MR a 70S & $7 / 60(\mathrm{MS})$ \\
Chihuahua & 111 & 98 & $\mathrm{~S}$ & 60S a 100S & $50 \mathrm{~S}$ a 100S & $6 / 40(\mathrm{~T})$ \\
Cuauhtémoc & 112 & 100 & $\mathrm{~S}$ & 70S a 100S & 30MS a 80S & $6 / 40(\mathrm{~T})$ \\
Ópalo & 115 & 107 & $\mathrm{~S}$ & 70S a 100S & $60 \mathrm{~S}$ a 100S & $6 / 30(\mathrm{~T})$ \\
\hline
\end{tabular}

$\mathrm{DAM}=$ días a madurez; $\mathrm{AP}=$ altura de planta; $\mathrm{RT}=$ roya del tallo; $\mathrm{RC}=$ roya de la corona; $\mathrm{TR}=$ trazas de resistencia; $\mathrm{R}=$ resistente; $\mathrm{S}=$ susceptible; $\mathrm{MR}=$ moderadamente resistente; $\mathrm{MS}=$ moderadamente susceptible; $\mathrm{T}=$ tolerante.

\section{Potencial de rendimiento}

En el Cuadro 2 se presenta la comparación de rendimiento de grano, así como el porcentaje de disminución de las variedades testigo con respecto a Jade. Esta nueva variedad promedio de las 105 evaluaciones presentó mayor rendimiento de grano promedio, con $2241 \mathrm{~kg} \mathrm{ha}^{-1}$, superando a las variedades testigo desde $4 \%$ (Turquesa) hasta $45 \%$ (Ópalo).

Cuadro 2. Rendimiento de grano y porcentaje de disminución de las variedades testigo respecto a la nueva variedad Jade en diferentes ambientes de temporal de 2007 a 2012.

\begin{tabular}{cccccccccc}
\hline \multirow{2}{*}{ Variedad } & \multicolumn{3}{c}{ General $(105)$} & \multicolumn{2}{c}{$\mathrm{AF}(21)$} & \multicolumn{2}{c}{$\mathrm{AI}(43)$} & \multicolumn{2}{c}{$\mathrm{AC}(41)$} \\
\cline { 2 - 9 } & $\left(\mathrm{kg} \mathrm{ha}^{-1}\right)$ & $(\% / \mathrm{J})$ & $\left(\mathrm{kg} \mathrm{ha}^{-1}\right)$ & $(\% / \mathrm{J})$ & $\left(\mathrm{kg} \mathrm{ha}^{-1)}\right.$ & $(\% / \mathrm{J})$ & $\left(\mathrm{kg} \mathrm{h}^{-1}\right)$ & $(\% / \mathrm{J})$ \\
\hline JADE & 2241 & & 3681 & & 2402 & & 1337 & \\
Turquesa & 2144 & -4 & 3652 & -1 & 2203 & -8 & 1312 & -2 \\
Teporaca & 2036 & -9 & 3384 & -8 & 2138 & -11 & 1239 & -7 \\
Obsidiana & 2028 & -10 & 3332 & -9 & 2141 & -11 & 1242 & -7 \\
Karma & 1914 & -15 & 3205 & -13 & 1993 & -17 & 1171 & -12 \\
Menonita & 1804 & -20 & 2784 & -24 & 1925 & -20 & 1176 & -12 \\
Chihuahua & 1780 & -21 & 2706 & -26 & 1886 & -21 & 1196 & -11 \\
Papigochi & 1682 & -25 & 2746 & -25 & 1631 & -32 & 1191 & -11 \\
Diamante & 1658 & -26 & 2615 & -29 & 1700 & -29 & 1126 & -16 \\
Cuauhtémoc & 1554 & -31 & 2486 & -32 & 1621 & -33 & 1008 & -25 \\
Avemex & 1516 & -32 & 2450 & -33 & 1515 & -37 & 1041 & -22 \\
Ópalo & 1224 & -45 & 2057 & -44 & 1143 & -52 & 883 & -34 \\
Tukey $(\alpha=0.05)$ & 473 & -22 & 908 & -21 & 566 & -25 & 390 & -14 \\
\hline
\end{tabular}

$\mathrm{AF}=$ ambientes favorables; $\mathrm{AI}=$ ambientes intermedios; $\mathrm{AC}=$ ambientes críticos; \%/J $=$ porcentaje de disminución respecto a la variedad Jade. 
Los ambientes de producción en donde se evaluó se clasificaron en críticos con precipitación aproximada menor de $300 \mathrm{~mm}$ durante el ciclo de producción, en intermedios con precipitación aproximada de 300 a $500 \mathrm{~mm}$ y en favorables con precipitación aproximada mayor a $500 \mathrm{~mm}$. Con base en dicha clasificación ambiental, Jade mostró rendimientos de $3681 \mathrm{~kg} \mathrm{ha}^{-1}, 2402 \mathrm{~kg}$ $\mathrm{ha}^{-1}$ y $1337 \mathrm{~kg} \mathrm{ha}^{-1}$ en ambientes favorables, intermedios y críticos, respectivamente, superando a las 11 variedades testigos en cada una de las condiciones de producción señaladas.

El rendimiento promedio por tipo de ambiente de producción con respecto al promedio de los 11 testigos en cada uno, indica que Jade en ambientes favorables los superó $21 \%$, en ambientes intermedios en $25 \%$ y en ambientes críticos en $14 \%$, lo que en su momento sería un indicativo que Jade, por su mayor resistencia a roya del tallo, tiene más ventajas sobre las variedades testigo en los ambientes intermedios y en los favorables, en donde prevalecen condiciones adecuadas para la incidencia de la enfermedad. En los ambientes críticos por lo general prevalecieron condiciones de sequía, en donde también Jade mostró mejor respuesta que la mayoría de los testigos.

Es importante indicar que en general, la productividad de Jade fue considerablemente superior al de las variedades Menonita (20\%), Chihuahua (21\%), Papigochi (25\%), Diamante (26\%), Cuauhtémoc (31\%), Avemex (32\%) y Ópalo (45\%), por lo que al sustituir tales variedades por la nueva variedad Jade, se logrará incrementar la productividad de avena en siembras de temporal $28 \%$ en promedio, con mayor impacto en ambientes donde se presenta mayor incidencia de roya del tallo.

\section{Áreas de recomendación de producción}

La nueva variedad Jade se recomienda para su siembra en ambientes críticos, intermedios y favorables de producción en las áreas de temporal en donde se produce avena durante el ciclo agrícola primavera-verano en los estados de Oaxaca, Puebla, Tlaxcala, Hidalgo, Estado de México, Querétaro, Guanajuato, Michoacán, Jalisco, Aguascalientes, Zacatecas, Durango y Chihuahua.

En general se recomienda como la mejor opción, junto con la variedad Turquesa, en cualquier ambiente de producción; es importante recomendar variedades con fondos genéticos diferentes para formar mosaicos genéticos que no permitan la evolución dinámica de las razas de roya del tallo, de tal manera que, junto con Jade y Turquesa, también se recomiendan las variedades Teporaca, Obsidiana y Karma. También Jade es recomendable, junto con Turquesa y Obsidiana, para siembras de otoño-invierno, en los estados en donde se produce este cereal.

\section{Agradecimientos}

Al INIFAP por el apoyo brindado a través del proyecto denominado: generación de tecnología para incrementar la productividad del cultivo de avena en la región centro de México.

\section{Literatura citada}

Espitia, R. E.; Villaseñor, M. H. E.; Tovar, G. R.; De la O Olán, M. y Limón, O. A. 2012. Momento óptimo de corte para rendimiento y calidad de variedades de avena forrajera. Revista Mex. Cienc. Agríc. 3(4):771-783. 
Leyva, M. S. G.; Espitia, R. E.; Villaseñor, M. H. E. y Huerta, E. J. 2004. Pérdidas ocasionadas por Puccinia graminis f. sp. avenae Eriks. y Henn., causante de la roya del tallo en seis cultivares de avena (Avena sativa L.) en los Valles Altos de México. Rev. Mex. Fitopatol. 22(2):166-171.

Mariscal, A. L. A.; Huerta, E. J.; Villaseñor, M. H. E.; Leyva, M. S. G.; Sandoval, I. J. S. y Benítez, R. I. 2011. Selección de genotipos de avena para la identificación de razas de roya del tallo. Rev. Mex. Cienc. Agríc. 2(4):593-600.

SIAP. 2017. Servicio de información y Estadística Agroalimentaria y Pesquera. www.siap.gob.mx. Villaseñor, M. H. E.; Espitia R. E.; Huerta E. J.; Osorio A. L. y López, H. J. 2009a. Turquesa, nueva variedad de avena para la producción de grano y forraje en México. Agric. Téc. Méx. 35(4):487-492.

Villaseñor, M. H. E.; Huerta, E. J.; Rodríguez; G. Ma. F.; Hortelano, S. R. R.; Martínez, C. E. y Espitia, R. E. 2009b. Programa nacional de mejoramiento genético de avena en México: historia y aportaciones. Reseña Histórica 66 años al servicio de México 1943-2009. SAGARPA-INIFAP-CIRCE-CEVAMEX. 33-43 pp. 